\title{
Reconciling Validity and Challenges of Patient Comfort and Understanding: Guidelines to Patient- Oriented Questionnaires
}

Catherine Hudon ( $\nabla$ catherine.hudon@usherbrooke.ca )

Université de Sherbrooke https://orcid.org/0000-0001-6140-9916

Alya Danish

Universite de Sherbrooke Faculté de Médecine et des Sciences de la Santé: Universite de Sherbrooke

Faculte de medecine et des sciences de la sante

Mireille Lambert

Centre intégré universitaire de santé et de services sociaux du Saguenay-Lac-Saint-Jean

\section{Dana Howse}

Memorial University of Newfoundland

\section{Monique Cassidy}

University of New Brunswick

\section{Olivier Dumont-Samson}

Universite de Sherbrooke Faculté de Médecine et des Sciences de la Santé: Universite de Sherbrooke Faculte de medecine et des sciences de la sante

Judy Porter

Patient Partners

\section{Donna Rubenstein}

Patient partners

\section{Véronique Sabourin}

patient partners

\section{Shelley Doucet}

University of New Brunswick

\section{Vivian R. Ramsden}

University of Saskatchewan

\section{Mathieu Bisson}

Universite de Sherbrooke Faculté de Médecine et des Sciences de la Santé: Universite de Sherbrooke Faculte de medecine et des sciences de la sante

\section{Charlotte Schwarz}

University of New Brunswick

\section{Maud-Christine Chouinard}


Université de Montréal Faculté des sciences infirmières: Universite de Montreal Faculte des sciences infirmieres

\section{Short report}

Keywords: Patient-reported outcome measure, Questionnaire, Patient engagement

Posted Date: April 6th, 2021

DOl: https://doi.org/10.21203/rs.3.rs-367076/v1

License: (c) (1) This work is licensed under a Creative Commons Attribution 4.0 International License. Read Full License 


\section{Abstract}

Background: Patient-reported outcomes measures (PROMs) are widely recognized as important tools for achieving a patient-centered approach in health research. While PROMs are subject to several stages of validation during development, even questionnaires with robust psychometric properties may pose problems for targeted patients. Challenges can rise for reasons of clarity, understanding, comfort, or the complexity of the patient's situation (e.g., their health needs). How can good practices in questionnaire development and validation on one hand, and challenges of accommodating patient comfort and understanding on the other hand be reconciled? Building on the experience of patient engagement in the PriCARE research program, this paper aims to propose: 1) steps to address challenges of patient comfort and understanding of the questionnaires and to reach consensus, and 2) patient-oriented guidelines for administrating the questionnaires.

Methods: Based on a participatory approach and the patient engagement framework in the Strategy for Patient-Oriented Research of the Canadian Institutes of Health Research, team members, including patient partners, worked together to discuss the problem, review the questionnaires, and come up with different solutions. Based on literature presenting similar processes in research projects, a working group was created to produce guidelines for administering the questionnaires. We present a step-by-step description of strategies used in PriCARE, to reconcile good research practices for using validated questionnaires and the challenges in questionnaire development related to patient comfort and understanding.

Results: This paper demonstrates how patient partners were engaged in PriCARE and integrated into the program's governance structure, the challenges they raised regarding the questionnaires, and how the challenges were addressed in a six-step approach: 1) Recognizing patient partner concerns, discussing these concerns, and reframing the challenges; 2) Detailing and sharing evidence of the validity of the questionnaires; 3) Evaluating potential solutions; 4) Searching literature for guidelines; 5) Creating guidelines; 6) Sharing and refining guidelines.

Conclusion: This six-step approach demonstrates how research teams can integrate patient partners as equal members, develop meaningful collaboration through recognition of individual experiences and expertise, and ensure the patient perspective is taken into consideration in questionnaire research, the development of data collection tools, and healthcare innovation in general.

\section{Background}

Patient-reported outcomes measures (PROMs) now are widely recognized as an important tool in the philosophy of a patient-centered approach to assess care. They highlight what really matters in the patient's life and can measure the impact of an intervention from the patients' perspective [1]. As stated by international guidance, PROMs questionnaires are subject to several stages of validation, such as identifying the conceptual framework of the measure and assessing validity and fidelity $[2,3]$. Despite 
recommendation from the scientific community to avoid the reformulation of questions after a PROM has been validated $[4,5]$, even questionnaires with robust psychometric properties may pose challenges for patients in terms of clarity, level of comfort completing the questionnaire, or the applicability of a generalized questionnaire to their complex situation. The meaning of a question may not always be clear to patients [6, 7]. Background demographic data collection may be perceived as a potential threat [8].

Questionnaires may be challenging for certain groups. For people with cognitive impairments, quantitative evaluation, comparison questions, abstract concepts, and generalised statements can be challenging [9]. The unique situation of patients with multiple chronic conditions is not always taken into account in the way questions are formulated [10]. Individuals belonging to some vulnerable populations may have difficulty understanding rating scales and mutually exclusive response choices or may find them too complex [11-14]. Rigid adherence when administering questionnaires to older people may inhibit the interviewer from interacting and engaging meaningfully with the respondent or responding to cues indicating distress or grief [13].

To overcome some of these challenges, Boynton, Wood and Greenhalgh [12] propose a guide to questionnaire research for "reaching beyond the white middle classes". For example, they suggest placing demographic questions at the end of the questionnaire to minimize the threat to participants and explaining clearly why this information is required and how it will be protected. They also suggest working closely with community representatives and concluded that the quality of social interactions during questionnaire administration undoubtedly influences responses.

How can good practices in questionnaire development and validation on one hand, and challenges of accommodating patient comfort and understanding on the other hand be reconciled? Building on the experience of the participatory PriCARE research program, this paper addresses this question and proposes 1) steps to address challenges of patient comfort and understanding of the questionnaires and to reach consensus, and 2) patient-oriented guidelines for administrating the questionnaires.

\section{Methods}

\section{Challenges with questionnaires: the example of the PriCARE research program}

The PriCARE research program $[15,16]$ was funded by the Canadian Institute of Health Research in the Strategy for Patient Oriented Research (SPOR) - Primary and Integrated Health Care Innovation (PIHCl) Network programmatic grants. The program's overarching goals are to 1) generate findings on the implementation of case management (CM) in primary care for individuals with chronic conditions and complex healthcare needs who frequently use healthcare services, and 2) implement an evidence-based intervention to improve care experience and outcomes and to guide policy decision-making. 
The PriCARE research program is a multiple-case embedded mixed- methods study with a participatory approach [17] conducted in five participating Canadian provinces of Newfoundland and Labrador, Nova Scotia, New Brunswick, Quebec, and Saskatchewan. One or two primary care clinics were recruited in each province to implement and evaluate the $\mathrm{CM}$ intervention among frequent users of healthcare services with chronic diseases and complex care needs. The intervention is detailed elsewhere [15]. To meet the inclusion criteria for the program, participants must be: over 18 years of age, living with at least one chronic condition (including mental health), have complex health care needs, heavy use of health care services according to professional judgment, and could benefit from the intervention. Patients with loss of autonomy, living in a long-term care residence, having a prognosis of less than one year, or unable to consent were excluded.

In coherence with the Strategy for Patient-Oriented Research of the Canadian Institutes of Health Research, the PriCARE team actively engages patient partners as equal team members in all research aspects [18]. A community of patient partners was formed, including six patient partners who work closely with the research team, and patient partners are involved in research steering committees. They were integrated into the program's governance structure to ensure their engagement in all research phases and to ensure a focus on their priorities. Patient partners were involved in all stages of the PriCARE research program: formulation of the research objectives, review of data collection tools, training of the case managers, recruitment of participants, program monitoring, data analysis and interpretation, and drafting of publications. One of the roles of patient partners was to represent the voice of patients with complex care needs.

\section{The patient questionnaires}

The PriCARE research program underwent evaluation employs a mixed-method data collection approach, including self-administered questionnaires. Based on a literature review, instruments were carefully selected to measure several variables, such as health literacy $[19,20]$, multimorbidity $[21,22]$, care integration [23, 24], self-management [25, 26], quality of life [27, 28] and psychological distress [29, 30]. Inclusion criteria were: a self-administered questionnaire with good psychometric properties that was available in English and French and included a low number of items. An additional file describes the selected instruments in more detail [see Appendix 1].

\section{Challenges faced}

Before completing data collection was initiated, patient partners reviewed the patient questionnaires, which was comprised of several pre-validated instruments [Appendix 1], and expressed reservations regarding the general administration of the questionnaires, as well as with specific questions. First, patient partners indicated that going through the questionnaires was an uncomfortable experience. They emphasized the personal nature of some of the questions, noting that certain questions could be triggering as a result of past negative health care experiences, and being asked to reflect on difficult topics. These topics included their ability to care for themselves, their experiences with physical or psychological distress as a result of multiple chronic illnesses, and mental health challenges. Patient 
partners emphasized the importance of establishing a relationship of trust between study representatives and participants by starting slowly, reviewing the purpose of the questionnaire, and easing into more difficult questions to help alleviate any possible discomfort.

Second, patient partners desired clarification on why participants were being asked such questions, and whether, or how, their responses would be beneficial. They felt that further justification was needed for particularly sensitive or invasive questions in order for participants to feel comfortable sharing their responses.

Third, patient partners found some of the questions confusing or found the wording difficult to understand. They felt that there were several ways to interpret these questions, and that participants would need assistance, as the response choices did not always reflect the type of answer participants would have thought to provide.

Fourth, patient partners felt that some of the questions were phrased in a way that presumed the respondent had a single health condition and did not adequately reflect the realities of living with multiple chronic conditions. For example, when considering a question concerning health literacy, patient partners noted that some of the respondent's illnesses may be well-managed by the participant, while others are may be more challenging and may be poorly less understood. Thus, patient partners therefore expected participants would find found it difficult for participants to answer questions which that did not account for addressed multiple, complex needs. Patient partners expressed interest in adding, removing, or revising some of the questionnaire items and felt that some of them seemed outdated. It is important to note that many patients suffer from multiple chronic conditions, often alongside mental health conditions and/or social vulnerabilities [19-21], which may interfere with their ability to complete a questionnaire [12].

\section{Results}

\section{Addressing the challenges of patient comfort and understanding of the questionnaires and reaching consensus}

In co-construction with patient partners, the PriCARE team engaged in a 6-step approach to address the above challenges.

Step 1: Recognizing patient partner concerns, discussing to better understand and acknowledge these concerns, and reframing the challenges

Patient partners first identified, examined, and discussed the challenges regarding the questionnaires and agreed on main concerns. Specifically, these concerns included the absence of an introduction to the questionnaires, misunderstanding of unclear certain words or phrases, lack of precision of certain 
imprecise and/or unnecessary questions, difficulty for participants with inappropriateness of some questions for participants with multiple conditions to complete the questionnaires, and problematic. They also provided critical feedback on sociodemographic questions, such as including a lack of consideration for gender diversity. Then, patient partner representatives presented their concerns to other members of the PriCARE team. The main difficulty was to reconcile the patients' concerns alongside the academic researcher requirements to maintain questionnaire validity. Through discussion and reflection, members of the PriCARE team recognized patient partners' concerns and determined how to proceed. The team agreed that a prudent first step was to present to the patient partners evidence of the questionnaires' validity and the degree to which patients were engaged in tool development (for example, whether is there any involvement in the tool development involved or cognitive interviewing with patients or other forms of patient consultation).

\section{Step 2: Detailing and sharing evidence for the validity of the questionnaires}

A table, similar to Appendix 1, detailing the evidence of the instruments' validity and supporting their use was presented to the PriCARE team, including patient partners, to support the discussion of potential solutions. For each variable, the PriCARE team questioned whether other instruments would be more appropriate. Literature searches were undertaken to identify potentially relevant instruments. At the end of this process, based on the criteria for selecting tools, the team, including patient partners, concluded that initial instruments were appropriate for the research program.

\section{Step 3: Evaluating potential solutions}

Despite the above mentioned concerns raised by the patient partners, the possibility of replacing or introducing significant modifications to the questionnaires was discarded as this could compromise the psychometric properties of instruments that had already been formally assessed for reliability and validity [11]. However, it was obvious that the PriCARE team could not use the self-administered questionnaires without introducing minor modifications, such as the inclusion of techniques to promote patient comfort and understanding. The PriCARE team favored the use of neutral statements and probes that would not affect the validity of the measures (for example, by saying: there are no right or wrong answers), interviewing techniques to encourage response to difficult questions (for example, by providing clarification to stimulate a response), and reducing the burden of the patient by considering some of the challenging aspects of the questionnaires (to reduce fatigue, consider sensitivity to triggers, distress) [31].

\section{Step 4: Searching the literature for guidelines}

The need to adapt questionnaire items for vulnerable and diverse populations has been noted in the literature [11], yet few publications discuss addressing this by creating guidelines to facilitate the administration of questionnaires. To develop their own guidelines, the PriCARE team searched the literature for academic publications: 1) providing information to develop guidelines; and 2) reporting patients' challenges with the completion of validated questionnaires. 
Boynton, Wood and Greenhalgh [12] propose that representing "disempowered and socially excluded groups, cross cultural issues, and participants whose physical and mental health may interfere with their ability to complete a questionnaire" may be addressed through careful administration of questionnaires in addition to training and support of research staff. The administration of questionnaires is seen as a social interaction that may be challenging for both the participant and the researcher [8], and as such, must be rethought to recognize and overcome their inherent bias towards white, well-educated populations, and to adapt them to the needs of larger segments of the population $[8,11]$.

The World Health Organization regularly develops guidelines for administration to accompany survey instruments [32]. One such example is the World Health Survey's "Guide to administration and question by question specifications," meant to accompany a survey instrument developed to compile comprehensive baseline information on the health of populations [31]. The guide is used as a training tool for interviewers when administering the questionnaires and contains instructions, interview guidelines, questionnaire conventions, and background information on each question and why it is asked. This manual's structure, the topics it covered, and the intent behind its development have proved useful in the development of similar guidelines for the PriCARE questions. The section covering the use of clarifications, probing techniques, and feedback as essential components of standard interviewing techniques have been particularly useful.

The decision was made to develop guidelines for the administration of the questionnaires that would respond to the challenges identified by patient partners and support researchers in considering the needs of vulnerable patients while introducing only minor modifications to the questionnaires.

\section{Step 5: Creating the guidelines}

A working group was formed including two postdoctoral researchers, two research assistants, and three patient partners to discuss modifications and create patient-oriented guidelines for administering the PriCARE questionnaires. Three meetings were held. The first was a brainstorming session to discuss the format and content of the guidelines in response to the challenges posed by the questionnaires and documented in prior meetings of the community of patient partners. In the second meeting, a first draft of the guidelines based on the World Health Survey's "Guide to administration and question by question specifications" was presented and discussed, and modifications to the questionnaires proposed by the patient partners were agreed upon. In the third meeting, a second draft of the guidelines and modified questionnaires was reviewed and prepared for presentation to the larger PriCARE team.

\section{Step 6: Sharing and refining the guidelines}

The working group presented the guidelines and questionnaires to the PriCARE team. Team members gave feedback, edited the text, and made suggestions in order to clarify the content of the guidelines. These suggestions were incorporated into the final version of the documents. This version was reviewed and approved by members of the PriCARE team. 


\section{Patient-oriented guidelines for administration of questionnaires}

A manual was created to guide the administration of the PriCARE questionnaires. The manual includes six sections. Section I introduces the PriCARE research program and the purpose of the questionnaires, with the objective to ensure the expertise of the researcher/interviewer is familiar with the overall research program. Section II outlines the roles and responsibilities of the researcher and includes training material on interviewing techniques and preparing to administer difficult or sensitive questions. Section III describes how to administer the questionnaires, including how to prepare the respondent and what to say to them before beginning the questionnaires. Section IV reviews the use of neutral probes, feedback and clarifications on an as-needed basis. Section $V$ includes a copy of the questionnaires with clarifications added beneath questions that patient partners had flagged as challenging. Section VI includes instructions on how to debrief the respondent after completing the questionnaires and end the session. The manual to guide the administration of the PriCARE questionnaires is presented in Appendix 2.

The primary modification to the PriCARE patient questionnaires was a context modification [11] where the questionnaires would no longer be self-administered by participants but would be completed with researchers in person or over the phone. Researcher administered questionnaires are intended to facilitate participant completion but are considered more labour intensive [8]. The responsibility to comprehend and work through challenging questions becomes shared between the participant and the researcher. The guidelines are thus intended to accompany the researcher who may need training and support in administering the questionnaires [8].

The second modification to the questionnaires was a format modification where clarifications were added to challenging questions in order to reduce respondent burden, and enhance comprehension and motivation to complete the questionnaires [11]. Clarifications are required in situations where the participant is unable to understand the question or the response choices, or expresses difficulty or confusion regarding a questionnaire item [31]. Short statements were added next to challenging questions, and interviewers were instructed in the guidelines only to use them after neutral probes or feedback failed to stimulate a response.

\section{Discussion}

\section{Process and guidelines adaptable for patient-oriented projects}

With the growing interest worldwide in PROMs [1, 33-35], optimizing patient understanding and comfort during the administration of questionnaires while ensuring validity and respect of psychometric properties becomes an important target to be achieved. Since the balance between these two aspects 
(comfort and validity) remains a challenge [11, 12, 14], the 6-step approach to reach consensus with patient partners and the guidelines presented in this paper will be useful for research teams.

The 6-step process could be shortened by maintaining steps 1 and 2 ('Documenting and discussing the problems,' and 'Detailing and sharing the evidence for the validity of the questionnaires'), and by adapting the 6 sections of our guidelines to any research project [see Appendix 3]. Section I of the guidelines could be modified to introduce the new project or program, the purpose of the questionnaires, with the objective of ensuring the expertise of the researcher/interviewer in the overall research program. Other sections of the guidelines could be slightly adapted to the new project. Section $V$ should be reviewed to include a copy of the questionnaires with clarifications added beneath questions that patient partners had flagged as challenging.

\section{Conclusions}

This six-step approach demonstrates how research teams can integrate patient partners as equal members into the research team and develop meaningful collaboration through recognition of individual experiences and expertise. Ultimately, incorporating these steps ensures the patient's perspective is expressed through questionnaire research, the development of data collection tools, and healthcare innovation in general.

\section{Abbreviations}

$\mathrm{CM}$

Case management

$\mathrm{PIHCl}$

Primary and Integrated Health Care Innovation

PROMs

Patient-reported outcomes measures

SPOR

Strategy for Patient Oriented Research

\section{Declarations}

Acknowledgement: We would like to thank members of the PriCARE team, the principal investigators, coinvestigators, coordinator and research assistants, case managers and patient partners.

Funding: This work is supported by the Canadian Institutes of Health Research (CIHR) - Operating Grant: SPOR PIHCI Network: Programmatic Grants (grant number 397896) and other partners such as Axe santé-Population, organisations et pratiques du CRCHUS, Centre de recherche du CHUS, Département de médecine de famille et médecine d'urgence (Université de Sherbrooke), Fondation de L'Université de Sherbrooke, Fondation de Ma Vie, Fonds de recherche du Québec en santé, Institut universitaire de 
première ligne en santé et services sociaux, Ministère de la santé et des services sociaux du Québec, New Brunswick Health Research Foundation, Nova Scotia Health Authority, Faculty of Medicine of Dalhousie University and Dalhousie Medical Research Foundation, Réseau-1 Québec, Saskatchewan Health Research Foundation and Université de Sherbrooke and Université du Québec à Chicoutimi.

Authors' contributions: Catherine Hudon and Maud-Christine Chouinard contributed to the PriCARE research program conception and design. Alya Danish led the different steps for the patient-oriented guidelines to administration of the questionnaire with the involvement of Dana Howse, Monique Cassidy, Olivier Dumont-Samson, Judy Porter and Donna Rubenstein. The first draft of the manuscript was written by Catherine Hudon

and Alya Danish and all authors commented on previous versions of the manuscript. All authors read and approved the final manuscript.

Ethics approval and consent to participate: Not applicable because no primary data were collected.

\section{Consent for publication: Not applicable.}

Competing interests: The authors declare that they have no competing interests.

Availability of data and material: The datasets supporting the conclusions of this article are included within the article and its additional files.

\section{References}

1. Weldring T, Smith SM. Patient-Reported Outcomes (PROs) and Patient-Reported Outcome Measures (PROMs). Health Serv Insights. 2013;6:61-8.

2. Reeve BB, Wyrwich KW, Wu AW, Velikova G, Terwee CB, Snyder CF, et al. ISOQOL recommends minimum standards for patient-reported outcome measures used in patient-centered outcomes and comparative effectiveness research. Qual Life Res. 2013;22:1889-905.

3. Health, USDo, Human Services FDACfDE, Research, Health USDo, Human Services FDACfBE, Research, et al. Guidance for industry: patient-reported outcome measures: use in medical product development to support labeling claims: draft guidance. Health Qual Life Outcomes. 2006,4:79.

4. Juniper EF. Validated questionnaires should not be modified. Eur Respir J. 2009;34:1015-7.

5. Floyd J, Fowler JR, Mangione TW. Standardized survey interviewing minimizing interviewer-related error. Newbury Park: Sage; 1990.

6. Conrad FG, Schober MF. Clarifying question meaning in a household telephone survey. Public Opin Q. 2000;64:1-28.

7. Schober MF, Conrad FG, Fricker SS. Misunderstanding standardized language in research interviews Applied Cognitive Psychology. 2004,18:169-88.

8. Boynton PM. Administering, analysing, and reporting your questionnaire. BMJ. 2004;328:1372-5. 
9. Beadle-Brown J, Ryan S, Windle K, Holder J, Turnpenny A, Smith N, et al. Engagement of people with longterm conditions in health and social care research: Barriers and facilitators to capturing the views of seldom-heard populations. Canterbury: Policy Research Unit in Quality and Outcomes of person-centred care (QORU); 2012.

10. Paterson C. Seeking the patient's perspective: a qualitative assessment of EuroQol. COOP-WONCA charts MYMOP Qual Life Res. 2004;13:871-81.

11. Stewart AL, Thrasher AD, Goldberg J, Shea JA. A framework for understanding modifications to measures for diverse populations. J Aging Health. 2012;24:992-1017.

12. Boynton PM, Wood GW, Greenhalgh T. Reaching beyond the white middle classes. BMJ. 2004;328:1433-6.

13. de Vries K, Leppa CJ, Sandford R, Vydelingum V. Administering questionnaires to older people: rigid adherence to protocol may deny and disacknowledge emotional expression. J Aging Stud. 2014;31:132-8.

14. Isaksson U, Santamäki-Fischer R, Nygren B, Lundman B, Åström S. Supporting the very old when completing a questionnaire: Risking bias or gaining valid results? Research on Aging. 2007;29:57689.

15. Danish A, Chouinard MC, Aubrey-Bassler K, Burge F, Doucet S, Ramsden VR, et al. Protocol for a mixed-method analysis of implementation of case management in primary care for frequent users of healthcare services with chronic diseases and complex care needs. BMJ Open. 2020;10:e038241.

16. Hudon C, Chouinard MC, Aubrey-Bassler K, Burge F, Doucet S, Ramsden VR, et al. Case management in primary care for frequent users of healthcare services with chronic diseases and complex care needs: an implementation and realist evaluation protocol. BMJ Open. 2018;8:e026433.

17. Hudon C, Bisson M-CC, Danish M, Karam A, Girard M. A, et al. Case study with a participatory approach: Rethinking pragmatics of stakeholder engagement for implementation research.. Annals of Family Medicine. Accepted.

18. Amirav I, Vandall-Walker V, Rasiah J, Saunders L. Patient and Researcher Engagement in Health Research: A Parent's Perspective. Pediatrics. 2017,140.

19. Chew LD, Griffin JM, Partin MR, Noorbaloochi S, Grill JP, Snyder A, et al. Validation of screening questions for limited health literacy in a large VA outpatient population. J Gen Intern Med. 2008;23:561-6.

20. Hudon É, Hudon C, Couture EM, Lavoie M, Lambert S, Lambert M, et al. Measuring health literacy in primary health care: Validation of a French-language version of a three-item questionnaire. In NAPCRG Annual Meeting. Maryland, USA; 2016.

21. Bayliss EA, Ellis JL, Steiner JF. Subjective assessments of comorbidity correlate with quality of life health outcomes: Initial validation of a comorbidity assessment instrument. Health Quality of life Outcomes. 2005;3:51.

22. Poitras M-E, Fortin M, Hudon C, Haggerty J, Almirall J. Validation of the disease burden morbidity assessment by self-report in a French-speaking population. BMC Health Service Research. 
2012;12:35.

23. King J, Gibbons E, Graham C, Walsh J. Developing measures of people's self-reported experiences of integrated care. Oxford: Picker Institute Europe \& University of Oxford; 2013.

24. Joober H, Chouinard MC, King J, Lambert M, Hudon E, Hudon C. The Patient Experience of Integrated Care Scale: A Validation Study among Patients with Chronic Conditions Seen in Primary Care. Int J Integr Care. 2018;18:1.

25. Battersby MW, Ask A, Reece MM, Markwick MJ, Collins JP. The Partners in Health scale: The development and psychometric properties of a generic assessment scale for chronic condition selfmanagement. Australian Journal of Primary Health. 2003;9:41-52.

26. Hudon E, Chouinard MC, Krieg C, Lambert M, Joober H, Lawn S, et al. The French adaptation and validation of the Partners in Health (PIH) scale among patients with chronic conditions seen in primary care. PLoS One. 2019;14:e0224191.

27. Cheak-Zamora NC, Wyrwich KW, McBride TD. Reliability and validity of the SF-12v2 in the medical expenditure panel survey. Qual Life Res. 2009;18:727-35.

28. Dauphinee SW, Gauthier L, Gandek B, Magnan L, Pierre U. Readying a US measure of health status, the SF-36, for use in Canada. Clin Invest Med. 1997;20:224-38.

29. Kessler RC, Andrews G, Colpe LJ, Hiripi E, Mroczek DK, Normand SL, et al. Short screening scales to monitor population prevalences and trends in non-specific psychological distress. Psychol Med. 2002;32:959-76.

30. Arnaud B, Malet L, Teissedre F, Izaute M, Moustafa F, Geneste J, et al. Validity study of Kessler's psychological distress scales conducted among patients admitted to French emergency department for alcohol consumption-related disorders. Alcohol Clin Exp Res. 2010;34:1235-45.

31. World Health Organization. Guide to administration and question by question. specifications Geneva: World Health Organization; 2002.

32. World Health Organization. Assessment of human resources for health. Survey instruments: World Health Organization; 2002.

33. Recommendations to OECD ministers of health from the high level reflection group on the future of health statistics. Strengthening the international comparison of health system performance through patient-reported indicators. OECD. https://www.oecd.org/els/health-systems/Recommendationsfrom-high-level-reflection-group-on-the-future-of-health-statistics.pdf. Accessed August 25th, 2020.

34. Greenhalgh J. The applications of PROs in clinical practice: what are they, do they work, and why? Qual Life Res. 2009,18:115 - 23.

35. Gleeson H, Calderon A, Swami V, Deighton J, Wolpert M, Edbrooke-Childs J. Systematic review of approaches to using patient experience data for quality improvement in healthcare settings. BMJ Open. 2016;6:e011907.

\section{Supplementary Files}


This is a list of supplementary files associated with this preprint. Click to download.

- Appendix120210323.docx

- Appendix220210325.docx

- Appendix320210325.docx 